\title{
Physiological and Chemical Studies on the Bioconversion of Glycyrrhizin by Aspergillus niger NRRL595
}

\author{
Abdel-Monem H. El-Refai, Lotfy A. R. Sallam, Hassaan A. El- Menoufy and Hala Abdel Salam Amin* \\ Chemistry of Natural and Microbial Products Department, National Research Center, Dokki, Cairo, Egypt \\ E-mail: halaamin2007@yahoo.com
}

Received 14 February 2011; Received in revised form 5 December 2011; Accepted 11 January 2012

\begin{abstract}
Glycyrrhizin (GL), the well-known sweet saponin of licorice, has been used as a food-additive and as a medicine. Its aglycone, glycyrrhetic acid (GA) showed antiinflamatory, antiulcer and antiviral properties. GA is now produced form GL by acid hydrolysis. However, it is difficult to obtain GA in a good yield by using this method, because many by-products are also produced. Screening of different microorganisms (13 bacteria, 2 yeasts and 23 fungi) for production of GA from GL revealed that Aspergillus niger NRRL 595 produced the highest yield of GA. The bioconversion of GL by $A$. niger NRRL 595 for $96 \mathrm{~h}$, followed by isolation and purification of the transformation products led to the separation of two conversion products, namely: GA and 3-oxo-GA. Confirmation of the identity of these products was established by determination of their Rf values, m.p., and IR, UV, MS and NMR spectra. The conditions for cultivation of this fungus with the maximum hydrolytic activity for the maximum yield of GA were investigated. Based on the results, $A$. niger NRRL 595 was cultivated with a medium composed of $1.75 \% \mathrm{GL}, 0.5 \%$ glucose, $0.8 \%$ corn steep liquor at $\mathrm{pH} 6.5$ at $32{ }^{\circ} \mathrm{C}$ for $96 \mathrm{~h}$. The cultivation of fungal cells under the latter conditions afforded GA and 3-oxo-GA in a yield of $65 \%$ and $22 \%$, respectively.
\end{abstract}

Keywords: Glycyrrhizin, glycyrrhetic acid, bioconversion, A. niger NRRL 595

\section{INTRODUCTION}

Glycyrrhizin (GL) as a triterpenoid saponin derived from the root of licorice (Glycyrrhiza glabra) which is one of the most popular traditional medical herbs. GL is composed of one molecule of glycyrrhetic acid (GA) as aglycone and two molecules of glucuronic acid (Hennell et al. 2008). GA has expectorant and antitussive properties. It is widely used as a flavoring agent and is frequently employed to mask the taste of bitter drugs such as aloe and quinine. Contemporary research has identified anti-inflammatory, antiulcer, antiallergic, antiviral, antibacterial, and hepatoprotective effects demonstrated systemically by GA (Bombardelli et al. 1989; Akao 2000; Shibata 2000). Hyperkalemia is a common life-threatening problem in hemodialysis patients. Because GA inhibits the enzyme $11 \beta$-hydroxy-steroid dehydrogenase II and thereby increases cortisol availability to the colonic mineralocorticoid receptor, it has the potential to lower serum potassium concentrations (Farese et al. 2009). Recent study has shown that GA exhibits many pharmacological activities. Here a new derivative of GA, biotinylated GA, was synthesized by introducing the biotin to the C-29 carboxyl of GA, and its antitumor effects were confirmed through tests on mouse B16 melanoma cells and BEL 7402 human hepatocarcinoma cells (Shi et al. 2009). Kalaiarasi et al. (2009) reported that administration of $18 \beta$-glycyrrhetinic acid is able to reduce hyperglycemia and hyperlipidemia related to the risk of diabetes mellitus.
In principle, GA could be obtained by acid hydrolysis of the natural saponin followed by multi-stages processes for the purification of the resulting aglycone (Mahran et al. 1973). However, it is difficult to obtain GA in a good yield by this method, because many by-products are also produced. On the other hand, it has been disclosed that an enzyme named glycyrrhizinic acid hydrolase from Aspergillus niger selectively hydrolyzed 3-O- $\beta-D-$ glucuronide linkage of $G L$ without cleavage of the terminal glucuronobiose bond to give GA and glucuronobiose ( $\beta$-Dglucuronyl-(1-2)-D-glucuronic acid) in a high yield without formation of glucuronic acid (Muro et al. 1986). The action of this enzyme was different from those of the $\beta$ glucuronidases so far reported (Akao et al. 1986; Kim et al. 1999). Hattori et al. (1985) studied transformation of $\mathrm{GL}$ and related compounds by bacterial strains isolated from human feces. They found that Ruminococous sp. P01-3 hydrolyzed GL to GA in a yield of $34 \%$ after incubation for $40 \mathrm{~h}$. Sakano \& Ohshima (1986a) studied the conversion of GA using Streptomyces sp. G-20 with the aim of preparing useful new products. The authors found that this culture converted GA into 22- $\alpha$-hydroxy-18 $\beta-G A$ (as a major product) and two minor hydrolyxlated derivatives of that later major product. In addition, the same authors Sakano \& Ohshima (1986b) found that Chainia antibiotica IFO 12, 246 converted GA and 22ahydroxy GA into three kinds of 3,4-seco-oleanane-type conversion products. Studies were made by El- Menoufy (1988) on 102 different microbial cultures and found that Saccharomycoposis lipolytica was the most active 
organism, it catalyses the conversion of crude GL to GA in a yield of $49.6 \%$. The capacity of 8 bacterial strains, isolated from soil, for the conversion of GL to GA was assayed. Pseudomonas saccharophila showed the highest $\beta$-glucuronidase activity and may be useful for industrial fermentation (Tanaka et al. 1990).

The biotransformation process was found to be markedly influenced by the fermentation conditions including medium composition (kind and concentration of nutrients, substrate/product concentration), incubation temperature, $\mathrm{pH}$ value, dissolved oxygen (Kuramoto et al. 1994; Kim et al. 1999; Akao 1999a). Thus El- Menoufy (1988) found that among six nutritive media differing in the nature and/or the concentration of some constituents, the one containing glycerol, peptone, yeast extract, meat extract and $\mathrm{KH}_{2} \mathrm{PO}_{4}$ was the most favorable for the formation of GA from GL with Saccharomycopsis lipolytica. The present study aims at the selection of the most potent microorganism able to hydrolyze GL to GA. The selected strain was cultivated under different physiological conditions to evaluate its ability to transform GL, in order to achieve the maximal conversion output.

\section{MATERIALS AND METHODS}

\section{Microorganisms}

Different microorganisms used in this work (Table 1) were donated by the Center of Cultures of Natural and Microbial Products Chemistry Department, National Research Center, Cairo, Egypt. Experimental cultures were inoculated with descendants from a single slant of pure experimental organisms on potato dextrose agar. All the slant cultures were stored in a refrigerator with regular transfer every month.

Types of media

Medium I (\%, w/v): GL, 1; glucose, 1 and corn steep liquor (CSL), 0.8; pH 5.7 (Kuramoto et al. 1994).

Medium II (\%, w/v): GL, 1; CSL, 0.8; yeast extract 0.4 ; malt extract, 2; pH 5.3.

Medium III (\%, w/v): Malt extract, 2; yeast extract, $0.4 ; \mathrm{pH}$ 5.3 (Abd-Elsalam 2000).

Medium IV (\%, w/v): GL, 1; CSL, 0.8; pH 5.7.

Medium V (\%, w/v): Glucose, 2; peptone, 0.5; meat extract, 0.3 ; dry yeast, $0.3 ; \mathrm{NaCl}, 0.5 ; \mathrm{CaCO}_{3}, 0.3 ; \mathrm{pH} 7$ (Sakano \& Ohshima 1986b).

Medium VI (\%, w/v): Glycerol, 1; peptone, 0.5; meat extract, 0.3 ; yeast extract, $0.3 ; \mathrm{K}_{2} \mathrm{HPO}_{4}, 0.5 ; \mathrm{pH} 7.4$ (Sakano \& Ohshima 1986a).

Medium VII (\%, w/v): Glycerol, 0.5; glucose, 0.5; peptone, 0.5; yeast extract, 0.3; meat extract, $0.3 ; \mathrm{KH}_{2} \mathrm{PO}_{4}, 0.75$; pH 5.6 (El- Menoufy, 1988).

Medium VIII (\%, w/v): GL, 1; glucose, 1; peptone, 0.5; yeast extract $0.3 ; \mathrm{KH}_{2} \mathrm{PO}_{4}, 0.1 ; \mathrm{MgSO}_{4.7} \mathrm{H}_{2} \mathrm{O}, 0.05$, pH 5.7 (Kuramoto et al., 1994).

Media III, V, VI, and VII were supplemented with $1 \% \mathrm{GL}$ $(w / v)$ in the experiment studying the effect of medium composition on GL bioconversion.
Table 1: Bioconversion of GL to GA by the experimental micro-organisms as traced visually*.

TLC conditions

\begin{tabular}{|c|c|c|c|}
\hline Microorganism & $\mathrm{GA}$ & Microorganism & GA \\
\hline Bacteria & & M. rasimosis & - \\
\hline Bacillus sphaericus & - & $\begin{array}{l}\text { Aspergillus niger } \\
\text { NRRL } 595\end{array}$ & + \\
\hline B. cereus & - & A. niger NRRL3 & + \\
\hline B. megaterium & - & A. niger 1 & + \\
\hline B. coagulans & - & A. niger 2 & + \\
\hline B. sp. 25 & - & A. niger 4 & + \\
\hline B. sp. 38 & - & A. neveus & - \\
\hline B. subtilus & - & A. fumigatus & - \\
\hline $\begin{array}{l}\text { Mycobacterium } \\
\text { luteus }\end{array}$ & + & A. flavus & - \\
\hline $\begin{array}{l}\text { Micrococcus sp. } \\
360\end{array}$ & - & A. terreus & + \\
\hline Micrococcus sp. $6 B$ & - & A. ochraceous & + \\
\hline $\begin{array}{l}\text { Staphylococcus } \\
\text { aureus }\end{array}$ & - & A. versicolor & - \\
\hline Escherichia coli & - & A. carneus & - \\
\hline Pumillus sp. E 601 & - & $\begin{array}{l}\text { Penicillium } \\
\text { auranticum }\end{array}$ & - \\
\hline Yeasts & & P. waksmanii & - \\
\hline Candida tropicalise & - & P. aurantigrism & - \\
\hline $\begin{array}{l}\text { Saccharomyces } \\
\text { cerevisiae }\end{array}$ & - & P. chrysogenum & - \\
\hline Fungi & & P. islandicum & - \\
\hline Mucor roxii & - & P. cyclopium & + \\
\hline M. pusillus & - & Fusarium solani & + \\
\hline
\end{tabular}

- Transformation medium (medium I) $(\%, w / v):$ GL, 1 ; glucose, 1, CSL, 0.8; pH 5.7.

- *The presence of GA was detected by TLC analysis ( $p$. $37)$ and sign. (+) denotes its formation, while sign. (-) means that the microorganism fails to produce GA.

Thin layer chromatography (TLC) was performed on silica gel plates, (Fluka, silica gel 60F-254, layer thickness 0.2 $\mathrm{mm}$ ) using the following solvent systems:

A. Chloroform - petroleum ether - acetic acid (6:6:1, v/v). B. Acetic acid - n-butanol - 1,2-dichloroethane - water) $(4: 1: 4:, 1 . v / v)$.

\section{Preparation of inocula}

For bacteria and yeasts, a suspension of each microbial strain ( $2 \mathrm{~mL}$ of $48 \mathrm{~h}$ old cultures) was used to inoculate aliquots of $50 \mathrm{~mL}$ of liquid medium composed of $(\%, w / v)$ glucose, 1; peptone, 0.5; yeast extract, 0.3; malt extract, 0.3; agar, 2; pH 6.5 (Volesky \& Philips 1995) dispensed in $250 \mathrm{~mL}$ Erlenmeyer flasks. The flasks were agitated on a reciprocal shaker at $200 \mathrm{rpm}$ at $30^{\circ} \mathrm{C} \pm 2$ for $24 \mathrm{~h}$. For fungi, aliquots of $50 \mathrm{~mL}$ of liquid growth medium III were dispensed in $250 \mathrm{~mL}$ Erlenmeyer flasks and sterilized. 
The sterilized medium was inoculated with $2 \mathrm{~mL}$ of spore suspension prepared by adding $5 \mathrm{~mL}$ sterile distilled water into 6 days old slant culture. The flasks were agitated on a rotary shaker $(200 \mathrm{rpm})$ at $30^{\circ} \mathrm{C}$ for $48 \mathrm{~h}$.

\section{Biotransformation process: Screening expermints}

The sterilized fermentation medium I (containing $1 \% \mathrm{GL}$ ) was inoculated with the standard volume of inoculum (2 $\mathrm{mL}$ each for bacteria and yeasts and $5 \mathrm{~mL}$ for fungi). The flasks were agitated on a reciprocal shaker $(200 \mathrm{rpm})$ at $30{ }^{\circ} \mathrm{C} \pm 2$ for $48 \mathrm{~h}$ in case of bacteria and yeasts and for $72 \mathrm{~h}$ in case of fungi.

\section{Biotransformation process using A. niger NRRL 595}

Unless otherwise stated, medium I (containing $1 \% \mathrm{GL}$ ) (Kuramoto et al. 1994) was used for GA production by $A$. niger. The medium was adjusted to $\mathrm{pH} 5.7$ using $\mathrm{NaOH}$ or $\mathrm{HCl}$ solution, dispensed in $50 \mathrm{~mL}$ portions in $250 \mathrm{~mL}$ flasks and sterilized by autoclaving at $121^{\circ} \mathrm{C}$ for $15 \mathrm{~min}$ at 1.5 atmospheric pressure. These flasks were inoculated with $5 \mathrm{~mL}$ of the preculture suspension. Then, the flasks were fixed on a rotary shaker at $200 \mathrm{rpm}$ and $30^{\circ} \mathrm{C}$ for 72 h.

Extraction and determination of the converted products

At the end of the transformation period, contents of each flask (cells and medium) were acidified with $1 \mathrm{~N} \mathrm{HCl}$ to $\mathrm{pH}$ 2. The reaction mixture was extracted twice with double its volume of ethyl acetate. Then, the organic layer was washed three times with water to get rid of any residual $\mathrm{HCl}$, dried over anhydrous sodium sulphate and evaporated to dryness in vacuo to give solid residue (test material) (Kim et al. 1999). The residue was dissolved in chloroform - methanol mixture (1:1) and mounted on TLC plates. The plate was first chromatographed for GA and 3oxo-GA with solvent system $A$, and secondly for $G L$ with solvent system B (Akao et al. 1991). GL, GA and 3-oxoglycyrrhetic acid (3-oxo-GA) were detected on TLC plates under UV light or by acid charring $\left(10 \% \mathrm{H}_{2} \mathrm{SO}_{4}, 110^{\circ} \mathrm{C}\right.$, $10 \mathrm{~min}$ ). These compounds were quantitatively analyzed with TLC scanner (Shimadzu CS-9000 dual wave-length flying spot, thin layer chromato-scanner, Japan) at $\lambda s$ $=250 \mathrm{~nm}$ and $\lambda r=400$, by using calibration lines obtained from authentic samples (Hattori et al. 1985).

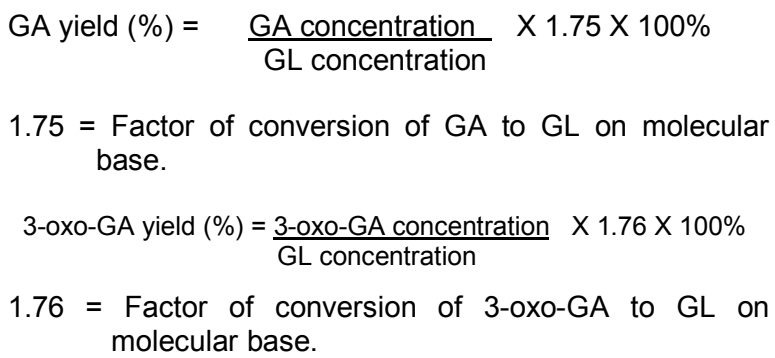
base.

3-oxo-GA yield $(\%)=3$-oxo-GA concentration $\times 1.76 \times 100 \%$ GL concentration

$1.76=$ Factor of conversion of $3-\mathrm{OxO}-\mathrm{GA}$ to $\mathrm{GL}$ on molecular base.

Separation and identification of the transformation products

Preparative TLC was carried out for separation of considerable amounts of transformed products. The test material dissolved in small volume of chloroform was applied to silica gel plates. The plates were eluted using solvent system $A$ and the resulted separated zones were scrapped off and extracted with chloroform. The solvent was filtered off and evaporated to dryness in vacuum. This leads to separation of two crude compounds I (150 mg) and II $(80 \mathrm{mg})$. Compounds were further purified separately on Sephadex LH-20 column (2.5 X 60), using methanol as an elution solvent. The purified transformed products I and II were then analyzed by infrared (IR), UV, FD-mass and NMR.

Compound I: It was identified as glycyrrhetic acid from its m.p., IR, UV, MS and NMR. It was obtained as colourless crystals $\left(110 \mathrm{mg}\right.$ ); m.p. $293-295^{\circ} \mathrm{C}$ (Reported m.p. 294296, Hattori et al. 1983); UV $\lambda_{\max }\left(\mathrm{CHCl}_{3}\right): 250 \mathrm{~nm}$; IR (potassium bromide) $\mathrm{cm}^{-1}: 3438(\mathrm{OH}), 1706(\mathrm{C}=\mathrm{O}), 1664$ (conjugated $\mathrm{C}=\mathrm{O}) 2875,2944,2968$ ( $\mathrm{CH}$ aliphatic); MS : $\mathrm{m} / \mathrm{z} 470\left(\mathrm{M}^{+}, 16.74 \%\right), 303$ (100\%), 262 (81\%), 248 (19 \%), 216 (17\%), 175 (49 \%), 135 (80\%); ${ }^{1} \mathrm{H}$ NMR (DMSO$\left.\mathrm{d}_{6}\right) \mathrm{ppm}: \delta 00.68,00.74,00.91,01.02,01.12,01.33$, 01.46 (each $\left.3 \mathrm{H}, \mathrm{s}, \mathrm{C}-\mathrm{CH}_{3}\right), \delta 03.04(1 \mathrm{H}, \mathrm{m}, \mathrm{CH}-\mathrm{OH}), \delta$ $05.44(1 \mathrm{H}, \mathrm{C}=\mathrm{CH}), 12.2(\mathrm{br}, 1 \mathrm{H}, \mathrm{OH}$, disappears after exchanged by $\mathrm{D}_{2} \mathrm{O}$ ); ${ }^{13} \mathrm{C}$-NMR: $\delta 016.0-055.3\left(25 \mathrm{SP}^{3}\right.$ carbons corresponding to $\left.C_{1}, C_{2}, C_{4}-C_{10}, C_{14}-C_{29}\right), \delta 076.6$ $\left(\mathrm{CH}-\mathrm{OH}\right.$, corresponding to $\left.\mathrm{C}_{3}\right), \delta 127.3,169.7$ ( $2 \mathrm{SP}^{2}$ carbons, $\mathrm{C}=\mathrm{C}$, corresponding to $\mathrm{C}_{12}, \mathrm{C}_{13}$ ), $\delta 177.7,199.0$ (2 SP2 carbonyl carbons, $\mathrm{C}=\mathrm{O}$, corresponding to $\mathrm{C}_{11}, \mathrm{C}_{30}$ ).

Compound II (3-oxo- glycyrrhetic acid): was obtained as colourless crystals $(60 \mathrm{mg}) ; \mathrm{mp} .305^{\circ} \mathrm{C}$ (Reported m.p. > 300, Hattori et al., 1983); UV $\lambda_{\max }\left(\mathrm{CHCl}_{3}\right): 250 \mathrm{~nm}$; IR (potassium bromide) $\mathrm{cm}^{-1}$ : $3312(\mathrm{COOH}), 1727,1682$ $(2 \mathrm{C}=\mathrm{O}), 1645$ (conjugated $\mathrm{C}=\mathrm{O}), 2875,2924,2968(\mathrm{CH}$ aliphatic) ; MS : m/z $468\left(\mathrm{M}^{+}, 47 \%\right), 453(25 \%), 440(27$ \%), 422 (17\%), 303 (94\%), 262 (93\%), 216 (27\%), 137 (19\%), 135 (100\%) ; ${ }^{1} \mathrm{H}-\mathrm{NMR}$ (DMSO-d $)$ ppm : $\delta 00.76$, 00.94, 00.98, 01.05, 01.07, 01.13, 01.33 (each 3H, s, C$\left.\mathrm{CH}_{3}\right), \delta 05.44(1 \mathrm{H}, \mathrm{s}, \mathrm{C}=\mathrm{CH}) ;{ }^{13} \mathrm{C}-\mathrm{NMR}: 015.8-060.5$ (25 SP $\mathrm{SP}^{3}$ carbons corresponding to $\mathrm{C}_{1}, \mathrm{C}_{2}, \mathrm{C}_{4}-\mathrm{C}_{10}, \mathrm{C}_{14^{-}}$ $\mathrm{C}_{29}$ ), $\delta$ 127.4, 171.1 (2 SP${ }^{2}$ carbons, $\mathrm{C}=\mathrm{C}$ corresponding to $\mathrm{C}_{12}, \mathrm{C}_{13}$ ), $\delta 178.4,199.0,216.1$ (3 $\mathrm{SP}^{2}$ carbonyl carbons, $\mathrm{C}=\mathrm{O}$, corresponding to $\mathrm{C}_{30}, \mathrm{C}_{11}, \mathrm{C}_{3}$, respectively).

\section{RESULTS AND DISCUSSION}

\section{Screening experiments}

The screening experiments were carried out with ultimate goal of selecting the most active GL transforming microorganism. Studies were made on 38 different microbial cultures (13 bacteria, 2 yeasts and 23 fungi) using $G L$ as a carbon source. These cultures manifested large variations in capacity to produce GA. Thus, while 
some cultures failed to perform the desired reaction (14mould fungal, 13 bacterial and 2 yeast cultures), the other experimental organisms could perform the desired reaction with the production of different amounts of GA, depending on the type of culture employed (Table 1). On the other hand, Mycobacterium luteus, $A$. niger NRRL 595, $A$. niger NRRL 3, A. niger $1, A$. niger $2, A$. niger 4, $A$. terreus, $A$. ochraceous, Penicillium cyclopium and Fusarium solani successfully converted GL to GA (Table 2).Under the experimental conditions employed, the tested $A$. niger NRRL 595 was proved to be the most potent microorganism that could hydrolyze GL leading to formation of promising amounts of $\mathrm{GA}$. It could transform about $18 \%$ of the added GL with the formation of about $1 \mathrm{mg} / \mathrm{mL}$ GA. In this connection, Muro et al. (1986) and Sasaki et al. (1988) reported that glycyrrhizinic acid hydorlase produced by $A$. niger GRM3 selectively hydrolyzed 3-O- $\beta$-D-glucuronide linkage of $G L$ to give the aglycon, GA and 2-O- $\beta$-D-glucuronyl-D-glucuronic acid (glucuronobiose). Recently, it was reported that GL biotransformation by $A$. niger included two stages: first, the two glucuronic acid residues at the C-3 position of GL were hydrolyzed to produce GA; and second, GA was oxidized and hydroxylated to compounds 1 and 2 . These were $\quad 7 \beta, 15 \alpha$-dihydroxy-3,11-dioxo-oleana-12-en-30-oic acid (1) and 15a-hydroxy-3,11-dione-oleana-12-en-30-oic acid (2) (Hongzhi et al., 2009).

Table 2: The amounts of GA produced by the promising tested microorganisms

\begin{tabular}{lcc}
\hline \multirow{2}{*}{ Microorganism } & \multicolumn{2}{c}{ GA out put } \\
\cline { 2 - 3 } & $\mathrm{mg} / 100 \mathrm{~mL}$ & $\begin{array}{c}\text { Conversion } \\
\%\end{array}$ \\
\hline Mycobacterium. luteus & 16.9 & 2.96 \\
Aspergillus niger NRRL & 101.3 & 17.73 \\
595 & 6.90 & 1.21 \\
A. niger NRRL 3 & 20.76 & 3.63 \\
$\quad$ A. niger 1 & 24.8 & 4.34 \\
A. niger 2 & 53.63 & 9.39 \\
A. niger 4 & 20.42 & 3.57 \\
A. terreus & 13.38 & 2.34 \\
A. ochraceous & 1.35 & 0.27 \\
Penicillium cyclopium & 8.66 & 1.52 \\
Fusarium solani & & \\
\hline
\end{tabular}

$\mathrm{GL}$ concentration $=1000 \mathrm{mg} / 100 \mathrm{ml}$ medium

Isolation and identification of the transformation products encountered after transformation of GL by A. niger NRRL 595.

Efforts have been imposed to isolate and confirm the identity of the encountered conversion products. Therefore, the experimental fungus was cultivated for $96 \mathrm{~h}$ in GL containing medium (namely medium I). Two conversion products, main product I $(70 \%)$ and minor product II (30\%) were isolated. The TLC analysis reveals that the main product I which has Rf 0.33 is glycyrrhetic acid (GA) and the other minor product II, whose $\mathrm{Rf}=0.68$ is 3-oxo-glycyrrhetic acid (3-oxo-GA). This preliminary identification was based on studying the TLC profile of products I and II as compared with authentic samples of the same compounds. The identification of the produced GA and 3-oxo-GA was furthermore confirmed by studying their IR, MS and NMR characteristics.

Table 3: ${ }^{1} \mathrm{H}$ NMR and ${ }^{13} \mathrm{C}$ NMR assignments of the isolated compounds

\begin{tabular}{|c|c|c|c|c|}
\hline \multirow[t]{2}{*}{ Carbon No. } & \multicolumn{2}{|c|}{ GA } & \multicolumn{2}{|c|}{ 3-OXo-GA } \\
\hline & ${ }^{1} \mathrm{H}$ & ${ }^{13} \mathrm{C}$ & ${ }^{1} \mathrm{H}$ & ${ }^{13} \mathrm{C}$ \\
\hline 1 & - & 38.6 & - & 40.8 \\
\hline 2 & - & 27.1 & - & 28.3 \\
\hline 3 & 3.04 & 76.6 & - & 216.1 \\
\hline 4 & - & 40.4 & - & 41.2 \\
\hline 5 & - & 54.1 & - & 54.0 \\
\hline 6 & - & 18.3 & - & 21.2 \\
\hline 7 & - & 32.1 & - & 33.9 \\
\hline 8 & - & 42.9 & - & 43.5 \\
\hline 9 & - & 61.1 & - & 60.5 \\
\hline 10 & - & 36.7 & - & 36.4 \\
\hline 11 & - & 199.0 & - & 199.0 \\
\hline 12 & 5.44 & 127.3 & 5.44 & 127.4 \\
\hline 13 & - & 169.7 & - & 171.1 \\
\hline 14 & - & 44.9 & - & 47.3 \\
\hline 15 & - & 25.7 & - & 26.0 \\
\hline 16 & - & 26.1 & - & 26.4 \\
\hline 17 & - & 31.5 & - & 31.8 \\
\hline 18 & - & 48.0 & - & 48.3 \\
\hline 19 & - & 40.6 & - & 43.3 \\
\hline 20 & - & 43.1 & - & 44.9 \\
\hline 21 & - & 30.3 & - & 31.5 \\
\hline 22 & - & 37.5 & - & 37.7 \\
\hline 23 & 0.68 & 27.8 & 0.76 & 28.7 \\
\hline 24 & 0.74 & 16.0 & 0.94 & 15.8 \\
\hline 25 & 0.91 & 16.2 & 0.98 & 18.3 \\
\hline 26 & 1.02 & 17.2 & 1.05 & 18.5 \\
\hline 27 & 1.12 & 23.0 & 1.07 & 23.2 \\
\hline 28 & 1.33 & 28.1 & 1.13 & 28.8 \\
\hline 29 & 1.46 & 28.4 & 1.33 & 30.8 \\
\hline 30 & 12.2 & 177.7 & - & 178.4 \\
\hline
\end{tabular}

The maximum absorption of the main conversion product (I) in the UV at 250 is attributed to the characteristic conjugated carbonyl in ring $\mathrm{C}$. IR spectrum displayed an absorption band at $3438 \mathrm{~cm}^{-1}(\mathrm{br}, \mathrm{OH})$ and two carbonyl absorption bands at 1706 and $1664 \mathrm{~cm}^{-1}$. Its mass spectrum shows a molecular ion peak appeared at $\mathrm{m} / \mathrm{z}$ $470(16.47 \%)$ which matched with the molecular formula of $\mathrm{GA}\left(\mathrm{C}_{30} \mathrm{H}_{46} \mathrm{O}_{4}\right)$. Its ${ }^{1} \mathrm{H}-\mathrm{NMR}$ (Table 3 ) shows 7 signals at $\delta$ 00.68-0146 ppm (s, $7 \mathrm{CH}_{3}$, corresponding to methyl groups of carbons $\left.\mathrm{C}_{23}-\mathrm{C}_{29}\right), \delta 03.04(\mathrm{~d}, 1 \mathrm{H}, \mathrm{CH}-\mathrm{OH}$, corresponding to $\left.\mathrm{C}_{3}\right), \delta 5.44(\mathrm{~s}, 1 \mathrm{H}, \mathrm{C}=\mathrm{CH}$, corresponding to $\mathrm{C}_{12}$ ). Furthermore, ${ }^{13} \mathrm{C}-\mathrm{NMR}$ (Table 3 ) shows $26 \mathrm{SP}^{3}$ carbon atoms corresponding to $\mathrm{C}_{1}-\mathrm{C}_{10}$ and $\mathrm{C}_{14}-\mathrm{C}_{29}$ and $4 \mathrm{SP}^{2}$ carbons corresponding to $\mathrm{C}_{11}, \mathrm{C}_{12}, \mathrm{C}_{13}$, $\mathrm{C}_{30}$. This proves the presence of one carbon attached to hydroxyl group $\left(\mathrm{C}_{3}\right)$ that recorded at $\delta 76.6$; two unsaturated carbon atoms $\left(\mathrm{C}_{12}, \mathrm{C}_{13}\right)$ that recorded at $\delta$ 127.3 and $\delta 169.7$, respectively; and two carbonyl carbon atoms $\left(\mathrm{C}_{11}, \mathrm{C}_{30}\right)$ which recorded at $\delta 199$ and $\delta 177$, respectively. Therefore, the structure of I was identified as 
glycyrrhetic acid. The spectral data was found to be in good agreement with those published (Hattori et al. 1983; Akao et al. 1991; Kim et al., 1999).

The IR spectrum of the minor conversion product (II) displayed a broad absorption band at $3312 \mathrm{~cm}^{-1}(\mathrm{COOH})$ and three carbonyl absorption bands at 1727, 1682 and $1645 \mathrm{~cm}^{-1}$. Its mass spectrum shows a molecular ion peak at $\mathrm{m} / \mathrm{z} 468(46.46 \%)$ which matches with the molecular formula of 3-oxo-GA $\left(\mathrm{C}_{30} \mathrm{H}_{44} \mathrm{O}_{4}\right)$. Its ${ }^{1} \mathrm{H}$-NMR (Table 3) shows 7 signals $\delta 00.76-01.33 \mathrm{ppm}\left(\mathrm{s}, 7 \mathrm{CH}_{3}\right.$, corresponding to methyl groups of carbons $\mathrm{C}_{23}-\mathrm{C}_{29}$ ), $\delta$ $05.44 \mathrm{ppm}$ ( $\mathrm{s}, 1 \mathrm{H}, \mathrm{C}=\mathrm{CH}$, corresponding to $\mathrm{C}_{12}$ ). In agreement, with this suggestion, the ${ }^{13} \mathrm{C}-\mathrm{NMR}$ spectrum of II (Table 3) shows $25 \mathrm{SP}^{3}$ carbon atoms corresponding to $\mathrm{C}_{1}, \mathrm{C}_{2}, \mathrm{C}_{4}-\mathrm{C}_{10}, \mathrm{C}_{14}-\mathrm{C}_{29}$ and $5 \mathrm{SP}^{2}$ carbon atoms corresponding to $C_{3}, C_{11}, C_{12}, C_{13}, C_{30}$. These findings reveal the presence of 3 carbonyl carbon atoms $\left(\mathrm{C}_{3}, \mathrm{C}_{11}\right.$, $\mathrm{C}_{30}$ ) recorded at $\delta 216.1,199.0$ and 178.4 , respectively and two unsaturated carbon atoms $C_{12}, C_{13}$ recorded at $\delta$ 127 and 171.1 respectively. Therefore the structure of II was identified as3-oxo- glycyrrhetic acid. In agreement with our results, Hattori et al. (1983) reported that GL was transferred into three metabolites (GA, 3-epi-GA and 3oxo-GA) by human intestinal flora. Also, 3-oxo-GA was obtained as a metabolite in the metabolism of GL by cultivation of rat gastrointestinal bacteria (Akao, 1997).

\section{Physiology of the transformation process}

\section{Suitability of the cultivation medium}

Results in Table 4 show that the composition of the fermentation medium greatly affected GL convertibility. Among tested media, medium I composed of (\%, w/v): GL, 1; glucose, 1; CSL, 0.8; pH 5.7 (Kuramoto et al. 1994) was proved to be the best fermentation medium that favored the hydrolysis of GL to GA and 3-oxo-GA (17.73, $7.4 \%$, respectively). This was accompanied by the highest total GL bioconversion estimate (25.19\%). Good GL bioconversion efficiency $(16.2 \%)$ was achieved on medium IV which is composed of $(\%, w / v)$ : GL, 1 ; CSL, 0.8 . These media (I \& IV) were characterized by the presence of GL as a carbon source and CSL as a sole nitrogen source. Similar results have also been reported by El-Menoufy (1988) who stated that, CSL could successfully replace peptone and meat extract. Thus, the superiority of medium I \& IV may be due to the presence of CSL which was not present in the other tested media.

\section{Effect of glucose, CSL, GL levels}

In examining effect of cultivation medium composition on the bioconversion process, different levels of either glucose, CSL or GL were used (Figures. 1, 2 and 3, respectively). The low level of glucose $(0.5 \%)$ supported maximum GL bioconversion activity (44.45\%), while increase of glucose level over the latter concentration resulted in a gradual decrease in GL bioconversion efficiency. The enhanced bioconversion efficiency in the presence of glucose might be due to increased growth of the fungus on this carbon source. However, increasing glucose concentration to $2 \%$ was accompanied by sharp decrease in the bioconversion efficiency, possibly because the cells preferred the easily oxidizable $d-(+)$ glucose as exclusive carbon source and repressed induction of GL hydrolyzing activity (Manosroi et al. 2003).

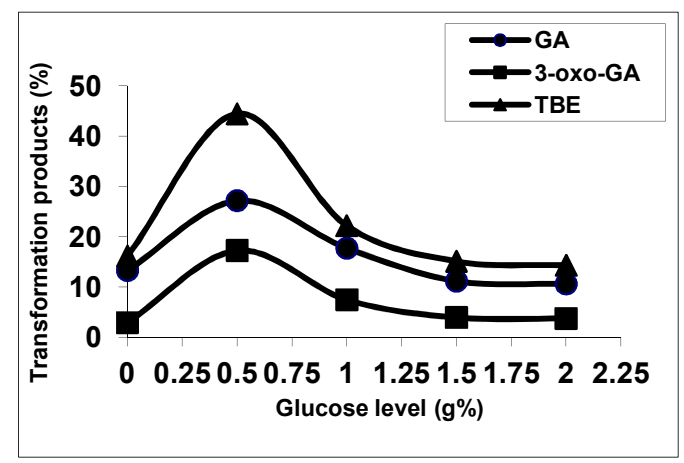

Figure 1: Effect of glucose level on GL bioconversion by A. niger NRRL 595

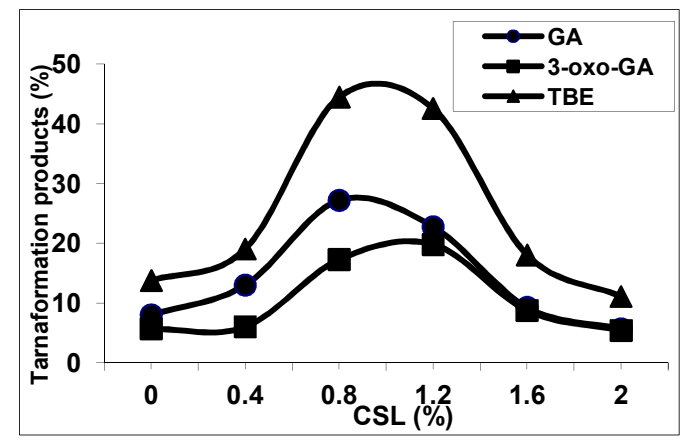

Figure 2: Effect of corn steep liquor level on $G L$ bioconversion by $A$. niger NRRL 595

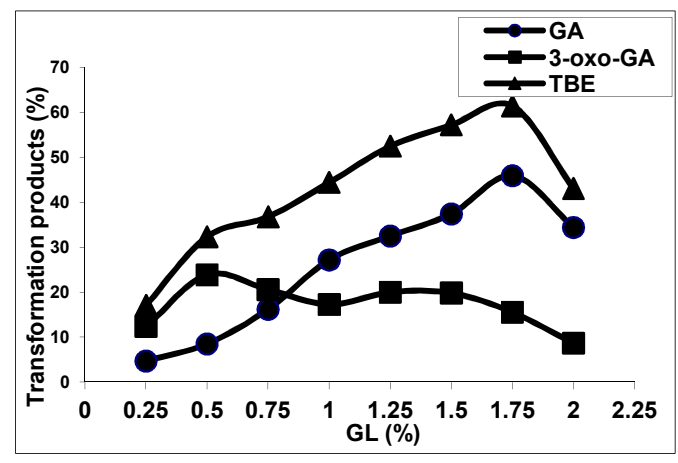

Figure 3: Effect of GL level on GL bioconversion by $A$. niger NRRL 595 
Table 4: Effect of composition of the fermentation medium on GL bioconversion by $A$. niger NRRL 595

\begin{tabular}{ccccc}
\hline \multirow{2}{*}{ Medium } & $\begin{array}{c}\text { Residual } \\
\text { GL }(\%)\end{array}$ & \multicolumn{2}{c}{$\begin{array}{c}\text { Transformation } \\
\text { products }\end{array}$} & TBE \\
\cline { 3 - 4 } & & GA (\%) & $\begin{array}{c}\text { 3-oxo-GA } \\
(\%)\end{array}$ & \\
\hline I & 64.9 & 17.73 & 7.46 & 25.19 \\
II & 93.9 & 0.82 & $0 . / 14$ & 0.96 \\
III & 92.8 & 1.86 & 0.33 & 2.19 \\
IV & 60.5 & 13.3 & 2.90 & 16.2 \\
V & 93.7 & 1.3 & 0.23 & 1.53 \\
VI & 92.9 & 1.75 & 0.3 & 2.05 \\
VII & 89.2 & 2.66 & 0.49 & 3.15 \\
VIII & 86.5 & 6.07 & 2.48 & 8.55 \\
\hline
\end{tabular}

Total bioconversion efficiency T.B.E $(\%)=$ GA yield $(\%)+$ 3-oxo-GA yield (\%)

Table 5: Bioconversion of GL by $A$. niger NRRL 595 as influenced by the initial $\mathrm{pH}$ value of the fermentation medium*

\begin{tabular}{|c|c|c|c|c|}
\hline \multirow{2}{*}{$\begin{array}{c}\text { Initial } \\
(\mathrm{pH})\end{array}$} & \multirow{2}{*}{$\begin{array}{c}\text { Residual } \\
\text { GL (\%) }\end{array}$} & \multicolumn{2}{|c|}{ Transformation products } & \multirow{2}{*}{$\begin{array}{r}\text { TBE } \\
(\%)\end{array}$} \\
\hline & & GA (\%) & $\begin{array}{c}\text { 3-oxo-GA } \\
(\%)\end{array}$ & \\
\hline 4 & 76.7 & 16.80 & 0.24 & 17.04 \\
\hline 4.5 & 70.2 & 20.06 & 5.28 & 25.34 \\
\hline 5 & 50.5 & 28.95 & 8.97 & 37.92 \\
\hline $5.7^{* *}$ & 31.3 & 45.91 & 15.55 & 61.45 \\
\hline 6 & 24.1 & 51.25 & 17.48 & 68.73 \\
\hline 6.5 & 22.3 & 51.45 & 18.25 & 69.70 \\
\hline 7 & 24.8 & 49.86 & 18.02 & 67.88 \\
\hline
\end{tabular}

and CSL, 0.8

- Transformation period: $72 \mathrm{~h}$

$-{ }^{\star \star}$ Control treatment: $\mathrm{pH}$ of the fermentation medium was adjusted to $\mathrm{pH} 5.7$

The influence of different CSL concentrations on GL bioconversion indicated that as the amount of CSL increased the GA yields were gradually increased and reached maximum $(27.18 \%)$ at $0.8 \%$ CSL level. On the other hand, the maximum 3 -oxo-GA yield $(19.8 \%)$ and total GL bioconversion efficiency $(47.5 \%)$ were recorded on using $1.2 \%$ CSL. Also, Kuramoto et al. (1994) found that CSL was effective for the hydrolysis activity. These findings may be of practical importance, since CSL is a cheap raw material. The steeping water had been reported to contain considerable amounts of sugars, nitrogenous compounds, organic acids (namely, lactic acid) amino acids and vitamins (Liggett \& Koffler 1948).

The effect of $G L$ level on the transformation process revealed that the bioconvertibility of $\mathrm{GL}$ increased from 17.1 to $61.45 \%$ by increasing GL concentration from 0.25 to $1.75 \%$, respectively. Maximum GA output (45.91\%) was recorded at $1.75 \% \mathrm{GL}$ level, while the highest yield of 3 -oxo-GA was recorded at $1.25 \% \mathrm{GL}$ level. These results suggest that a fermentation medium composed of (\%, w/v) glucose 0.5 , CSL 0.8 , and GL 1.75, affords the best bioconversion estimates of $\mathrm{GL}$, together with the formation of the highest yield of GA. In most cases, increase of GA is accompanied by increase of 3-oxo-GA. This may be explained by the enzymic reversibility between GA and 3-oxo-GA, which has been demonstrated by some investigators (Hattori et al. 1983, 1985; Akao 1997, 1999b). These authors suggested that, the equilibrium may be displaced in the direction of oxidation or reduction of GA or 3-oxo-GA according to fermentation conditions.

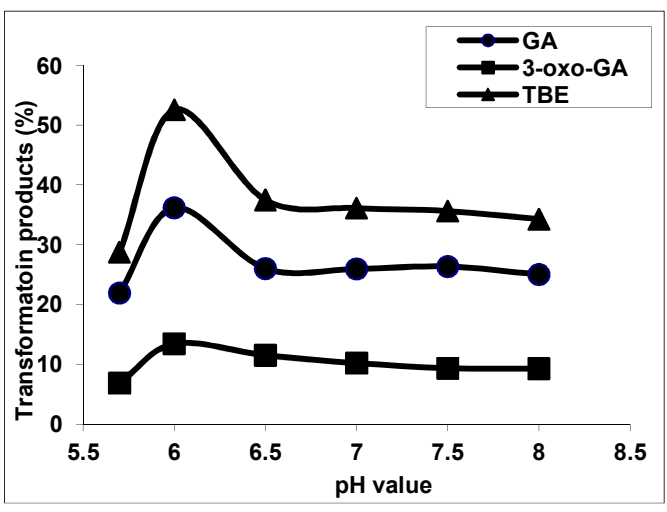

Figure 4: Bioconversion of GL by $A$. niger NRRL 595 grown on the fermentation medium adjusted with phosphate buffer at different $\mathrm{pH}$ values

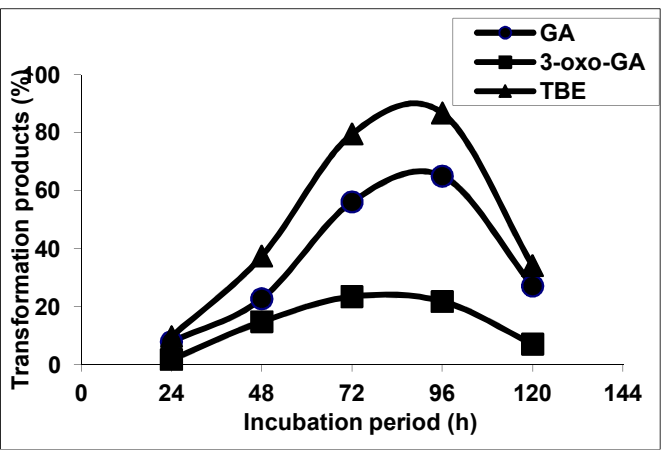

Figure 5: Effect of incubation period on GL bioconversion by $A$. niger NRRL 595

\section{$\mathrm{pH}$ value relation}

Control of $\mathrm{pH}$ value of the fermentation medium (initially or with buffer solutions) is an essential factor for the efficient enzymatic hydrolysis of GL by $A$. niger NRRL 595 . The results presented in Table 5 show that high conversion activities of GL were maintained within $\mathrm{pH}$ range of 6-7, however the bioconversion process was markedly retarded at $\mathrm{pH}$ values below 5.7. These findings support the data presented by El-Menoufy (1988) and Akao (1997) who reported that the enzymatic hydrolysis of $G L$ is 
difficult in the acidic medium. Maximum GA and 3-oxo-GA yieldsof 51.45 and $18.25 \%$, respectively, corresponding to total bioconversion efficiency of $69.7 \%$, were recorded at initial $\mathrm{pH}$ of 6.5. In agreement with the present results, Akao (1997) found that $\mathrm{GL}$ was hydrolyzed in the rat intestinal tract between $\mathrm{pH} 6$ and 7. However, Akao et al. (1987), El-Menoufy (1988), Akao (1999a) and Kim et al. (1999) reported that the optimal $\mathrm{pH}$ value of $\mathrm{GL} \beta$ glucuronidase activity was 5.6.

It is noteworthy to mention that, relatively good activities $(52.57 \%)$ were recorded on performing the transformation process in phosphate buffered medium (pH 6) (Figure 4). However, the capacity of the microorganism to transform GL was greatly suppressed in acetate buffered medium at $\mathrm{pH}$ values from 3.6 to 5.6 , indicating the deleterious effect of the buffer constituents (data not shown).

\section{Effect of incubation temperature}

Concerning the effect of different incubation temperatures on GL bioconversion by $A$. niger NRRL 595, results show that relatively high bioconversion yields were obtained at temperature range of $30-35{ }^{\circ} \mathrm{C}$ (data not shown). Maximum GA output was obtained at $30{ }^{\circ} \mathrm{C}$. The optimum temperature for production of $\mathrm{GA}$ differs according to the used strain. For example, the intestinal human bacteria acted optimally when incubated at $37{ }^{\circ} \mathrm{C}$ (Hattori et al. 1983). However, Muro et al. (1986) had cultivated A. niger GRM3 at $27{ }^{\circ} \mathrm{C}$ to achieve good conversion of GL to GA.

\section{Transformation time course}

The capacity of the experimental organism to transform GL proved to be markedly affected by transformation period (Figure 5). Consumption of $\mathrm{GL}$ and the total bioconversion efficiencies were increased with the increase of the incubation period. Maximal yield of GA $(65 \%)$ was obtained after $96 \mathrm{~h}$ of incubation, with an obvious decline thereafter. However, the highest yield of 3-oxo-GA (23.43\%) was recorded after $72 \mathrm{~h}$. Moreover, the maximal total conversion value $(86.78 \%)$ was achieved after $96 \mathrm{~h}$ with consumption of about $90 \%$ of the added GL. During the different phases of the transformation process, GA was proved to be the major product. Hattori et al. (1985) reported that, GL was transformed into three metabolites (GA, 3-epi-GA, 3-oxo$\mathrm{GA}$ ) by human intestinal flora. The reaction seems to include hydrolysis of GL to GA and glucuronic acid and reversible transformation of $G A$ to 3-epi-GA via a metabolic intermediate (3-oxo-GA) by the intestinal flora. They also demonstrated that, GL could not be converted to the three metabolites mentioned above by the action of a single species of bacterium but could be converted by the cooperative action of bacteria responsible for different steps of the metabolic pathways (Hattori et al. 1985). A fairly large number of bacteria showed $\beta$-glucuronidase activity to hydrolyze $G L$ to $G A$, while a moderate number of the bacteria had activity to reduce 3-oxo-GA to either GA or 3-epi-GA (Hattori et al. 1983; 1985; Akao 1997).

\section{CONCLUSION}

Aspergillus niger NRRL 595 was screened and selected on the basis of its ability to hydrolyze GL producing high yield of GA. Maximum total conversion value of $86.78 \%$ was obtained using production medium composed of (\%, w/v): $1.75 \mathrm{GL}, 0.5$ glucose, 0.8 corn steep liquor; $\mathrm{pH}$ 6.5; with the consumption of about $90 \%$ of the added GL. The medium was inoculated with $15 \%(\mathrm{v} / \mathrm{v})$ inoculum and incubated at $30{ }^{\circ} \mathrm{C}$ for $96 \mathrm{~h}$. Under these optimal conditions, GA yield became about 3 times higher than 3oxo-GA yield ( $65 \%$ and $22 \%$, respectively) and the cells bioconversion efficiency increased from 25.19 to $86.78 \%$.

\section{ACKNOWLEDGEMENTS}

The Authors thank Prof. Dr. N. A. Hassan, Dr. A.E.M. Gaafar, Central Laboratory Services, National Research Centre, Cairo, Egypt, for IR, MS,NMR measurements and the Center of Cultures of Chemistry of Natural and Microbial Products Department, National Research Center, Cairo, Egypt.

\section{REFERENCES}

Abd Elsalam, I. S. (2000). Physiological, biochemical and dynamical studies on cyclosporine A production. Ph. D. Thesis, Faculty of Science, Tanta University, Tanta, Egypt.

Akao, T. (2000). Effects of glycyrrhizin and glycyrrhetic acid on the growth, glycyrrhizin [beta]-Dglucuronidase and 3 [beta]-hydroxysteroid dehydrogenase of human intestinal bacteria. Biological and Pharmaceutical Bulletin 23, 104-107.

Akao, Ta. (1997). Localization of enzymes involved in metabolism of glycyrrhizin in contents of rat gastrointestinal tract. Biological and Pharmaceutical Bulletin 20(2), 122-126.

Akao, Ta. (1999a). Purification and characterization of glycyrrhetic acid mono-glucuronide $\beta-D$ glucuronidase in Eubacteirum sp. GLH. Biological and Pharmaceutical Bulletin 22(1), 80-82.

Akao, Ta. (1999b). Influence of various bile acids on the metabolism of glycyrrhizin and glycyrrhetic acid by Ruminococcus sp. PO1-3 of human intestinal bacteria. Biological and Pharmaceutical Bulletin 22(8), 787-793.

Akao, Ta, Akao, Te and Kobashi, K. (1987). Glycyrrhizin $\beta$-D-glucuronidase of Eubacteirum sp., from human intestinal flora. Biological and Pharmaceutical Bulletin 35(2), 705- 710.

Akao, Ta, Akao, Te, Hattori, M, Namba, T and Kobashi, K. (1986). 3ß-Hydroxysteroid dehydrogenase of Ruminococcus sp. from human intestinal bacteria. Journal of Biochemistry (Tokyo) 99, 1425-1431.

Akao, Te, Akao, Ta, Hattori, M, Yamamoto, K. and Namba, T. (1991). Hydrolysis of glycyrrhizin to $18 \beta-$ glycyrrhetyl, monoglucuronide by lysosomal $\beta$-Dglucuronidase of animal livers. Biochemical and Pharmacology 41, 1025-1029. 
Bombardelli, E., Curd, S. B., Della Loggia, R., Del Negro, P., Tubaro, A. and Gariboldi, P. (1989). Anti-inflammatory activity of 18-[beta]-glycyrrhetinic acid in Phytosome[R] form. Fitoterapia 60, 29-37.

El-Menoufy, H. A. (1988). Microbiological transformation of some natural products. Ph. D. Thesis, Faculty of Science, Cairo University, Giza, Egypt.

Farese, S., Kruse, A., Pasch, A., Dick, B., Frey, B. M., Uehlinger, D. E. and Frey, F. J. (2009). Glycyrrhetinic Acid Food Supplementation Lowers Serum Potassium Concentration in Chronic Hemodialysis Patients. Kidney International 76(8), 877-884.

Hattori, M., Sakamoto, T., Kobashi, K. and Namba, T. (1983). Metabolism of Glycyrrhizin by human intestinal flora. Journal of Medical Plant Research 48, 38-42.

Hattori, M., Sakamoto, T., Yamagishi, T., Sakamoto, K., Konishi, K., Kobashi, K. and Namba, T. (1985). Metabolism of glycyrrhizin by human intestinal flora. II. Isolation and characterization of human intestinal bacteria capable of metabolizing glycyrrhizin and related compounds. Chemical and Pharmaceutical Bulletin 33(1), 210-217.

Hennell, J. R., Lee, S., Khoo, C. S., Gray, M. J. and Bensoussan, A. (2008). The determination of glycyrrhizic acid in Glycyrrhiza uralensis Fisch. ex DC. (Zhi Gan Cao) root and the dried aqueous extract by LC-DAD. J Pharmaceut Biomed Anal 47(3), 494500.

Hongzhi, H., Heshui, Y., Jie, Z., Liping, K., Bing, F., Xinbo, S., Baiping, M. (2009). Biotransformation of glycyrrhizin by Aspergillus niger. Biocatalysis and Biotransformation 27 (2), 90-95.

Kalaiarasi, P., Kaviarasan, K. and Pugalendi K. V. (2009). Hypolipidemic activity of $18 \beta$-glycyrrhetinic acid on streptozotocin-induced diabetic rats. European Journal of Pharmacology 612, 93-97

Kim D H, Lee S W, Han M J. (1999). Biotransformation of glycyrrhizin to $18 \beta$-glycyrrhetinic acid-3-O- $\beta-D$ glucuronide, by Streptococcus LJ-22, a human intestinal bacterium. Biological and Pharmaceutical Bulletin 22(3), 320-322.

Kuramoto, T., Ito, Y., Oda, M., Tamura, Y. and Kitahata S. (1994). Microbial production of glycyrrhetic acid-3O-mono- $\beta$-Dglucuronide from glycyrrhizin by Cryptococcus magnus MG-27. Bioscience, Biotechnology, and Biochemistry 58(3), 455-458.

Liggett, R. W. and Koffler, H. (1948). Corn steep liquor in microbiology. Bacteriological Reviews 12, 297.

Mahran, G. H., Balbaa, S. I., El-Hossary, G. A. and Selim, M. A. (1973). Isolation, identification and estimation of glycyrrhizin from Glycyrrhiza glabra L. growing in Egypt. Bulletin Faculty Pharmacy Cairo University 12(1), 71-80.

Manosroi J, Sripalakit P, Manosroi A. (2003). Factors affecting the biotransformation of chlormadinone acetate to delmadinone acetate. Journal of Molecular Catalysis B: Enzymatic 23, 37 - 42 .
Muro, T., Kuramoto, T., Imoto, K. and Okada, S. (1986). Purification and some properties of glycyrrhizinic acid hydrolase from, Aspergillus niger GRM3. Agriculture Biological Chemistry 50(3), 687-692.

Sakai, T., Kamezawa, S. and Kobashi, K. (1988). Aflatoxin inhibitor in herbal drugs. Proceedings of the Japanese-Association-of Supplement No. 1, 77-78.

Sakano, K. and Ohshima, M. (1986a). Structures of conversion products formed from $18 \beta$-glycyrrhetinic acid by Streptomyces sp. G-20. Agriculture Biological Chemistry 50(3), 763-766.

Sakano, K. and Ohshima, M. (1986b). Microbial conversion of $18 \beta$-glycyrrhetinic acid and $22 \alpha$ hydroxy-18 $\beta$-glycyrrhetinic acid by Chainia antibiotica. Agriculture Biological Chemistry 50(5), 1239-1245.

Shi, J., Xiao, J. and Wei, D. (2009). Synthesis of biotinylated 18ß-glycyrrhetinic acid and its effect on tumor cells activity. Medicinal Chemistry Research 18 (7), 538-544.

Shibata, S. (2000). A drug over the millennia: Pharmacognosy, chemistry, and pharmacology of licorice. Yakugaku Zasshi 120, 849-862.

Tanaka, M., Anzai, Y., Kato, F. and Koyama Y. (1990). Isolation of bacterial strains, which hydrolyze glycyrrhizin and produce glycyrrhizic acid, from soil. Journal of Pharmacobiodyn 13(6), 361-366.

Volesky, B. and May-Philips H. A. (1995). Biosorption of heavy metals by Saccharomyces cerevisiae. Applied Microbiology and Biotechnology 42, 797-806. 